\title{
Diseño e implementación de un prototipo de vivienda domótica basado en las plataformas arduino y android
}

Fecha de recepción: 11 de agosto de 2014

Fecha de aprobación: 2 de octubre de 2014

Pp.115-132
Camila Sánchez*

Alejandra Mesa** Carolina Manrique *** Herbert Calderón ${ }^{* * * *}$

Luis Cobo ${ }^{* * * * *}$

Rubén Dorado******

Camilo Mejia*******

\section{RESUMEN}

Este artículo presenta el desarrollo de un prototipo de casa inteligente de bajo costo como una experiencia en área de la domótica. El objetivo principal es reportar el proceso y la metodología que podrían ser usados por otros grupos que tengan como propósito un proyecto similar. Este reporte incluye fallos y aciertos clave que se tuvieron durante los diferentes procesos: planeación, diseño y ejecución del prototipo.

\section{Palabras ClaVe}

Domótica, arduino, android, electrónica, mecánica y programación.

\footnotetext{
* Estudiante del programa de Ingeniería de Producción, Universidad EAN

** Estudiante del programa de Ingeniería de Sistemas, Universidad EAN.

${ }^{* * *}$ Estudiante del programa de Ingeniería de Sistemas, Universidad EAN.

**** Estudiante del programa de Ingeniería de Producción, Universidad EAN.

***** Ph.D. en Ingeniería, Universidad de los Andes y en Génie Informatique, École

Polytechnique de Montréal. Magister en Ingeniería de Sistemas y Computación, Universidad de los Andes.

****** Magister en Ciencias de la Computación, Universidad de Tokyo. Pregrado en Ingeniería de Sistemas, Universidad Nacional de Colombia.

******* Magíster en Ingeniería Mecánica, Universidad Nacional de Colombia. Pregrado en Ingeniería Mecánica, Universidad Nacional de Colombia.
} 


\section{Design and Implementation of a Prototype of a Demotic Housing Based on Arduin and Android Platforms}

\section{Abstract}

This article aims at developing a prototype of low- cost-intelligent house as an experience in the demotic area which could be controlled by mobile devices. Its main objective is to report the process and methodology to be used by other groups having the same project with the same characteristics. This report describes key success and failures which were revealed through the processes of planning, design and execution of the prototype.

\section{KEY WORDS}

Demotic, arduin, android, electronic, mechanics, programming

\section{Conception et réalisation d'un prototype de domotique basé sur les plates-formes Arduino et Android}

\section{RÉSUMÉ}

Cet article présente la création d'un prototype de maison intelligente à bas coût dont la domotique serait contrôlée par des dispositifs mobiles. L'objectif principal de l'article est de rendre compte du processus et de la méthodologie pouvant être utilisés par d'autres groupes de recherche ayant un projet aux caractéristiques similaires. Ce rapport inclus les conclusions et réalisations principales révélées lors des processus de planification, conception et mise en place du prototype.

\section{Mots-clés}

Domotique, arduino, android, électronique, mécanique et programmation 


\section{Desenho e implementação de um protótipo de casa plataformas de automação baseados em Arduino e Android}

\section{ReSUMO}

Este trabalho apresenta o desenvolvimento de um protótipo de casa inteligente de baixo custo como uma experiência na área da domótica que pode ser controlado por meio de dispositivos móveis. O principal objetivo deste trabalho é relatar o processo e a metodologia que poderiam ser usados por outros grupos que tenham como objetivo um projeto com características similares. Este relatório inclui os principais acertos e falhas que ocorreram durante os diferentes processos: planejamento, desenho e execução do protótipo.

\section{PalaVRas-CHaVe}

Domótica, arduino, android, mecânica e programação. 


\section{Introducción}

E I problema tratado en este trabajo es el desarrollo de una maqueta que representa una casa inteligente, con algunos componentes automatizados. Esta área se conoce como domótica, término que define la automatización de hogares utilizando componentes electrónicos que aportan elementos de seguridad, gestión, comunicación y optimización de recursos a las viviendas (Brush et al., 2011). Algunos ejemplos de las funcionalidades finales que se podrían implementar son: un sistema automático de luces que se encienden y apagan si hay alguna persona presente, una nevera que automáticamente haga una lista de víveres necesarios y los pida a los proveedores cuando se estén acabando o un sistema que dé aviso a la policía cuando un ladrón esté intentando entrar a la casa.

Este artículo presenta el producto final del Semillero de Investigación en Robótica de la Facultad de Ingeniería de la Universidad EAN, y describe el proceso realizado para obtener una maqueta de una casa que tiene algunos elementos domóticos. Específicamente, los elementos son tres: luces, una puerta y un ventilador que se controlan por medio de sensores de acuerdo con unas reglas específicas. La primera regla establece que si hay alguna persona en el lugar, las luces deberían estar prendidas. Otra dice que el ventilador debería prenderse si está haciendo mucho calor, dependiendo de un umbral previamente definido. Además, el prototipo tiene un componente adicional: el uso de un dispositivo móvil con el que es posible controlar todos los elementos automáticos.

Una característica importante de este proyecto radica en que se inició con un equipo de trabajo integrado por docentes y estudiantes, algunos con experiencia en temas de automatización y otros completamente nuevos. Aunque el objetivo principal del proyecto era desarrollar un prototipo a escala que representara una casa y que tuviera componentes de domótica, también era importante que los nuevos integrantes comprendieran todos los conceptos involucrados, mientras este se venía desarrollando. Otro objetivo importante era que el proyecto debía hacerse utilizando una 
metodología documentable y, por lo tanto, replicable; es decir, debía tener una serie de pasos metódicos que permitieran replicar la experiencia el número de veces que fuera necesario.

La mayoría de trabajos previos se basan en experiencias de construcción de prototipos puntuales. Por ejemplo, en Gauger, Minder, Marrón, Wacker y Lacheman (2008), se pueden encontrar varios ejemplos de prototipos de redes inalámbricas de sensores-actuadores probados experimentalmente y aplicados a la automatización del hogar. El trabajo de Vassev, Hinchey y Nixon (2010), reporta experimentos con prototipos usando ASSL (Ajax Secure Service Layer) para redes domóticas, mientras que en Irwin et al. (2011), se presentan resultados en protocolos de monitoreo usando diferentes prototipos de prueba. Otros trabajos se dedican a tratar las implicaciones de la aplicación de la domótica de forma tanto práctica como filosófica. Por citar algunos ejemplos, desde el ámbito filosófico, Randall (2003), analiza las implicaciones sociales, problemas y posibles cambios que podrían presentarse en la sociedad en los años futuros, debido al avance de la tecnología y su uso para automatizar hogares. Otro estudio relacionado que vale la pena citar es el de Woodruff, Agustín y Foucault (2007), en el que se hace el análisis del uso de la automatización de hogares y su implicación religiosa, tomando como sujeto de estudio a 20 familias norteamericanas.

Por otro lado, otro tipo de estudios se enfocan en áreas más prácticas como el de la arquitectura. Por ejemplo, Rodden y Benford (2003), analizan los cambios en cuanto a diseño debido a la inclusión de la tecnolugía domótica; o ecoriómicos (Rode, Toye, \& Blackwell, 2005), en los que se analizan los futuros cambios relacionados con la programación de las nuevas tecnologías, por el cambio en las interfaces finales de usuario que posiblemente se requerirán gracias a la inclusión de las nuevas tecnologías domóticas. Finalmente, vale la pena mencionar el proyecto "Villa Domótica", presentado en Mateos, González, Poo, García y Olaiz (2010), en el que queda claro que la utilización de prototipos de "casas automáticas" es de gran ayuda para la enseñanza de técnicas de automatización aplicadas a la domótica. 
Este trabajo no sólo presenta un prototipo de una casa a escala con características domóticas, resultado de un proceso de investigación, sino que aún más importante, da a conocer el proceso metodológico fácilmente reproducible. Cada uno de los pasos mostrados presentan, además, una serie de consideraciones relacionadas con la experiencia a manera de fallos y aciertos.

Asi mismo, en el artículo se encuentra la descripción de la metodología usada, fase por fase en la cual se incluyen los detalles de la experiencia, se describen detalles de la implementación final y por último, las conclusiones.

\section{Descripción del proyecto}

El proyecto inició con el objetivo de desarrollar un prototipo de una casa que fuera controlada por elementos electrónicos, a manera de casa inteligente. De esta manera, cada uno de los integrantes del grupo aprendería las bases de los elementos electrónicos, lógicos y físicos que se usan para controlar los componentes domóticos. Luego de varias reuniones se definió un objetivo concreto con el que se pudiera empezar a trabajar e, igualmente, se pudiera definir una metodología de trabajo. Se decidió hacer una casa que tuviera los mínimos elementos: baño, cocina, una sala y una habitación principal (ver Figura 1). Uno de los grandes aciertos iniciales fue tratar de definir el proyecto a la mayor precisión. Paralelamente, se estaban trabajando dos proyectos similares que se empezaron con mayor rapidez. 
Figura 1. Esquema inicial de la maqueta.

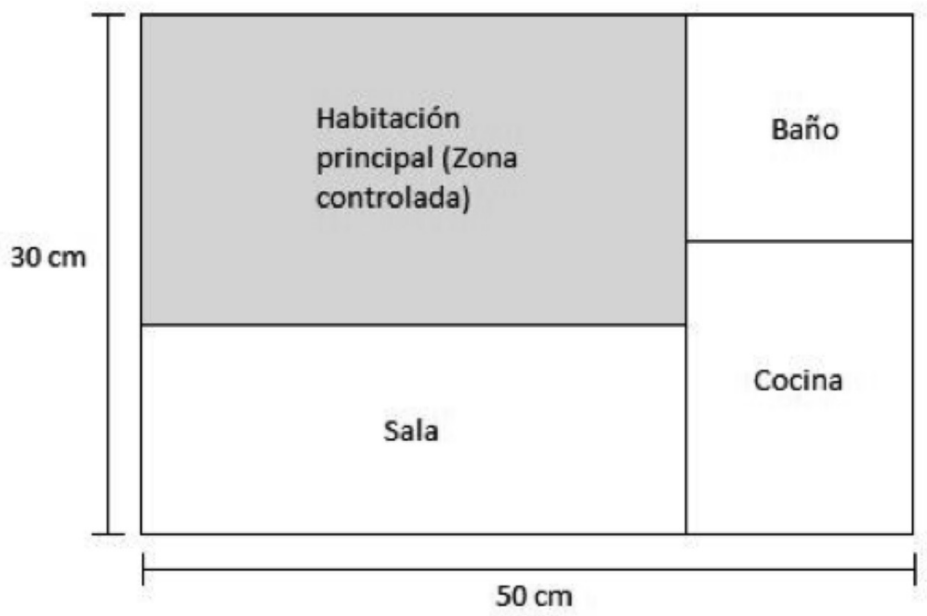

Fuente. Elaboración propia de los autores.

Igualmente, luego de las sesiones iniciales se acordó el uso de tres elementos domóticos: manejo de luces, una puerta y una ventana automatizada. Además, cada uno de estos elementos debería poderse controlar de manera remota con un dispositivo móvil.

\section{Metodología}

Una vez definido el objetivo así como el equipo de trabajo, se decidió concretar una metodología de trabajo clara con la que se pudiera minimizar el riesgo de fracaso. Algunos integrantes tenían experiencia en el manejo de proyectos de diferente naturaleza, entre otros, desarrollo de componentes electrónicos y proyectos de tipo industrial y de desarrollo de Software. Como particularidad interesante en este punto del proyecto, los expertos propusieron metodologías de trabajo relacionadas, por lo que se analizaron las ventajas y desventajas de algunas de estas para definir la que se tendría en cuenta finalmente. Luego de discutir diferentes posibilidades bastante interesantes, como por ejemplo, el uso de metodologías rápidas o el uso de metodologías de desarrollo de Software (Jacobson, Booch, \& Rumbaugh, 1999), se decidió utilizar una metodología que tuviera una serie de pasos bien definidos y que 
permitiera trabajar por fases. Además, esta metodología debería tener una etapa de planeación importante en la que se definiera la parte física, es decir, la maqueta, de una manera clara. Esto, debido a que una vez estuviera construida, se convertiría en la principal limitante física del proyecto. Haciendo un símil con un proyecto de la vida real, es posible añadirle elementos domóticos a una casa, siempre y cuando la parte física lo permita. Por ejemplo, si una puerta queda mal ubicada podría ser un inconveniente en el paso de su automatización incurriendo en costos adicionales.

Luego de la discusión, se seleccionó la siguiente metodología basada en fases:

- Fase 1: planeación.

- Fase 2: diseño y construcción de la maqueta.

- Fase 3: diseño e implementación de los módulos.

- Fase 4: integración.

A continuación se explican cada una de las fases mencionadas.

\subsection{Planeación}

La fase 1, llamada "Fase de planeación", define los detalles del producto final de manera minuciosa, tales como, las características físicas de la maqueta, las posiciones de los sensores y actuadores y la lógica general de la casa.

Esta fase fue de una importancia crítica, en conjunto con la fase de construcción de la maqueta, y se constituyó, en gran parte, en el éxito del proyecto, más aún, teniendo en cuenta que la parte física, es decir la maqueta, era la cadena vertebral del mismo y estaba combinada con las diferentes funcionalidades.

Para dar inicio a esta fase, primero se definió a manera de plano el tipo de casa por construir, visto desde arriba. Se decidió hacer una casa de un piso que tuviera 50 por $30 \mathrm{~cm}$ de área, con cuatro habitaciones, cuyos elementos domóticos serían: luces, una puerta y un ventilador; así mismo, que una sola zona de la casa tuviera los elementos de automatización. 
Estas definiciones fueron críticas en la fase posterior de elaboración, o sea planear la maqueta, y en especial para dejar los espacios necesarios para los sensores y actuadores.

La principal conclusión a la que se llegó con respecto a esta etapa se relaciona con el tiempo invertido, el cual fue valioso para todo el proyecto en general.

Las tareas que se tuvieron en cuenta y que están relacionadas con factores clave para lograr el resultado final son las siguientes:

- Definir de manera detallada el producto final y sus limitaciones.

- Definir de antemano cada uno de los elementos automatizados.

- Tener en cuenta los materiales y relacionarlos con costos.

- Definir un equipo de trabajo, identificando las fortalezas individuales de cada integrante.

Como principal desacierto por evitar en futuros proyectos de este tipo, está el de dar inicio a la siguiente fase con algunas variables sin definir. Por ejemplo, si no se definen los espacios para los motores y sensores, con seguridad se tendrán problemas a la hora de poner estos elementos en la maqueta. Es importante especificar cada uno de los elementos del producto final, para planear con detalle su elaboración.

\subsection{Diseño y construcción de la maqueta}

Una vez se tuvo una idea clara de los elementos, se empezó la construcción del componente posiblemente más importante del proyecto, la maqueta. denominada como "Fase de diseño y construcción de la maqueta". En esta fase se diseñó un plano con los detalles necesarios para construcciones, de una manera tal, que cumpliera con los objetivos funcionales. Este paso fue crítico para el proyecto, ya que limitaba los espacios y las posibilidades de automatización de elementos que se quisieran implementar; por ejemplo, en esta experiencia se diseñó el esquema vinculando cada uno de los tres componentes. Sin embargo, al diseñar la puerta automatizada no se tuvo en cuenta el tamaño exacto del motor actuador y en la etapa final, se tuvo que modificar parte de la maqueta. 
Como listado de acciones importantes durante esta fase, se proponen las siguientes:

- Tener en cuenta un espacio para los componentes: sensores, actuadores, el circuito, central de control y el cableado.

- Hacer planos con medidas detalladas.

- Relacionar cada componente automatizado con su posición física.

\subsection{Diseño e implementación de módulos}

En la fase 3, "Fase de diseño e implementación de módulos", se diseñaron componentes domóticos a nivel de electrónica y un componente de lógica que contiene toda la programación del control. A los componentes se les llamó módulos para definir una parte del proyecto separable y construible de manera independiente. Esto se hizo por tres razones: posibilitar la construcción de algunas partes de manera paralela, asignando módulos a los diferentes integrantes del grupo; facilitar la explicación del funcionamiento de los elementos a los integrantes no experimentados y posibilitar la experimentación con los sensores y actuadores sin tener todo el ruido de todo el proyecto.

Se definieron cuatro módulos principales, tres relacionados con una funcionalidad y un cuarto módulo de lógica de control. Cada uno de los módulos se explica a continuación:

- Módulo de luces: simula la funcionalidad de las luces inteligentes como ahorro de energía; sólo prende las luces si hay alguna persona que las utilice. De manera técnica se desarrolló con luces simuladas por LEDs y un sensor de infrarrojo. Si el sensor capta la presencia de alguna persona, entonces, el sistema prende las luces.

- Módulo de ventilador: la maqueta tiene la funcionalidad de un ventilador inteligente y automático; se activa solo cuando se supera un nivel de temperatura definido por el usuario. Para hacer este módulo se utilizó un ventilador y un sensor de temperatura. 
- Módulo de puerta: este módulo simula una puerta inteligente; se abre automáticamente si alguien quiere entrar a la sala. Para su implementación, se usó un servo-motor y un sensor infrarrojo.

- Módulo de lógica y comunicación: tiene como función controlar todos los módulos de acuerdo con una lógica de control. Como uno de los objetivos era que todos los componentes pudieran ser controlados de manera remota, este módulo revisa el estado de cada uno y de acuerdo con este modifica el actuador relacionado con el módulo, llamado lógica de tres estados, el cual se explica más adelante. El objetivo de crear un módulo de esta manera era posibilitar las pruebas con algunos componentes de forma individual o poder integrar los componentes uno a uno, incrementalmente.

Una particularidad importante del proyecto fue el diseño de una lógica a la que se llamó "lógica de tres estados", para poder controlar cada componente de manera remota. Teniendo en cuenta esta lógica, cada módulo, excepto el de control, tiene uno de tres estados posibles: apagado manual, encendido manual y encendido automático. Cuando el componente está en el estado apagado manual, quiere decir que el usuario decidió apagarlo, ignorando la lectura del sensor asociado. Por ejemplo, el usuario apaga la luz aunque alguna persona esté en la habitación. El encendido manual funciona de manera similar; se puede encender el sistema aunque la lectura del sensor y la lógica del sistema lleven a otra acción. Por ejemplo, prender el ventilador así esté haciendo frío. Finalmente, en el tercer estado, el componente se rige de acuerdo con una lógica automática que está controlada por las lecturas de los sensores.

El funcionamiento, a manera de programación lógica, puede verse en el ¡Error! No se encuentra el origen de la referencia, en el que las líneas cinco a la 13 muestran el comportamiento de cada uno de los componentes. 


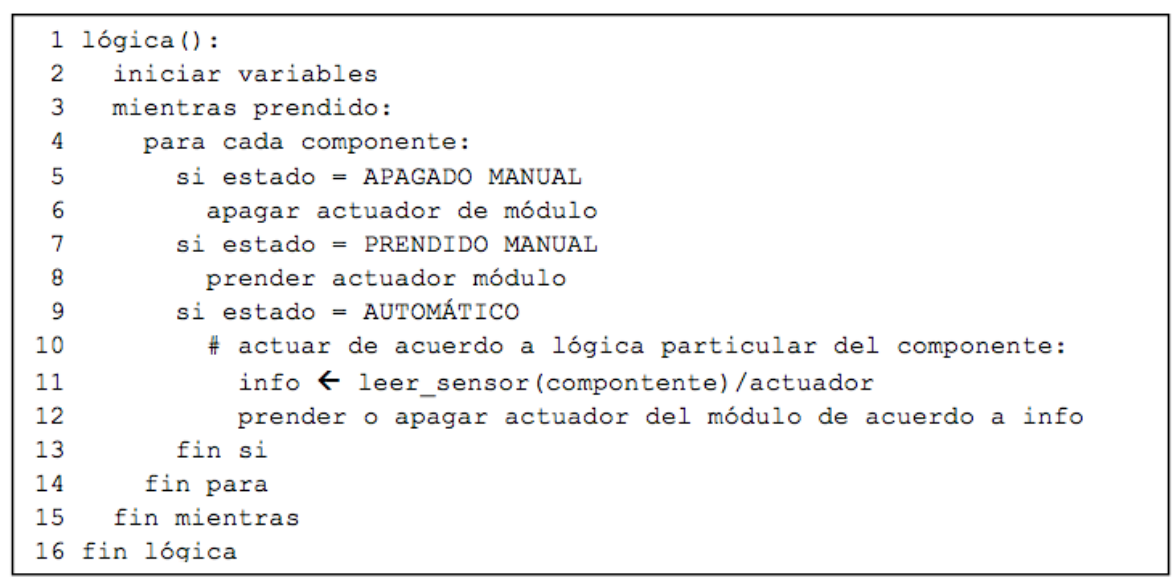

\subsection{Integración}

En la última fase, "Fase de integración", se combinaron todos los componentes domóticos con la maqueta para obtener el producto final. En este punto se pudieron ver los beneficios de trabajar con esta metodología, al igual que observar algunas fallas clave.

El propósito de esta fase era tomar cada uno de los módulos e ir adicionándolos uno a uno y de esta manera, tener un punto de inicio funcional y posibilitar la adición de cada uno de los componentes de manera gradual. En otras palabras, poder trabajar con un prototipo o maqueta inicial totalmente funcional y un módulo también probado. Si al integrar el módulo con toda la maqueta se presentaba algún error, entonces analizar de manera separada las partes involucradas y así, simplificar el problema general.

El proceso de integración se inició instalando el módulo de lógica de control a la maqueta. Luego, se fueron instalando cada uno de los otros módulos al proyecto. 


\section{Prototipo final}

Para la realización de este prototipo de casa inteligente, se decidió utilizar el microcontrolador Arduino, muy popular en el medio académico e industrial para el desarrollo de proyectos en automatización, robótica y electrónica en general. Según se establece en McRoberts (2013), Arduino es un microcontrolador diseñado para hacer más accesible para el público en general el proceso de utilizar dispositivos electrónicos en proyectos multidisciplinarios. El Hardware consiste de una "plaqueta" open-source diseñada alrededor del microntrolador AVR Atmel de 8 bits. Junto con este microcontrolador, el Arduino viene también con un compilador con un lenguaje de programación basado en el lenguaje $\mathrm{C}$ y un ambiente de desarrollo para construir los programas para esta plataforma.

El prototipo de "Casa inteligente" incorporó múltiples funciones que permitió llevar a la práctica interesantes aspectos de Hardware y Software de la domótica. A continuación, se presenta el esquema eléctrico final del prototipo de la "Casa inteligente". Se puede notar que el microcontrolador Arduino es el "cerebro" del proyecto. De allí se desprenden una serie de sensores y actuadores que tienen el objetivo de "darle vida" a la casa. Como se puede observar, hay una serie de sensores análogos conectados al microntrolador:

- Un sensor de temperatura, ubicado en la habitación principal de la casa, a partir de cuya información entrará en funcionamiento el ventilador cuando la temperatura rebase un cierto valor.

- Un sensor infrarrojo, de presencia, a partir de cuyas mediciones se encenderán o apagarán los leds de la casa.

También hay dentro de la casa, pero conectado a un puerto digital del microcontrolador, un sensor de ultrasonido ubicado sobre la puerta principal (ver Figura 3), cuya misión es detectar la presencia de personas en esta y activar un servomotor para abrir y cerrar tal puerta. 
El prototipo se completó con un transmisor inalámbrico Bluetooth (conectado al puerto serial del microcontrolador), que permite controlar diversos parámetros del prototipo de forma remota, ya sea desde un teléfono inteligente o desde un computador.

Figura 2. Esquema eléctrico de la maqueta

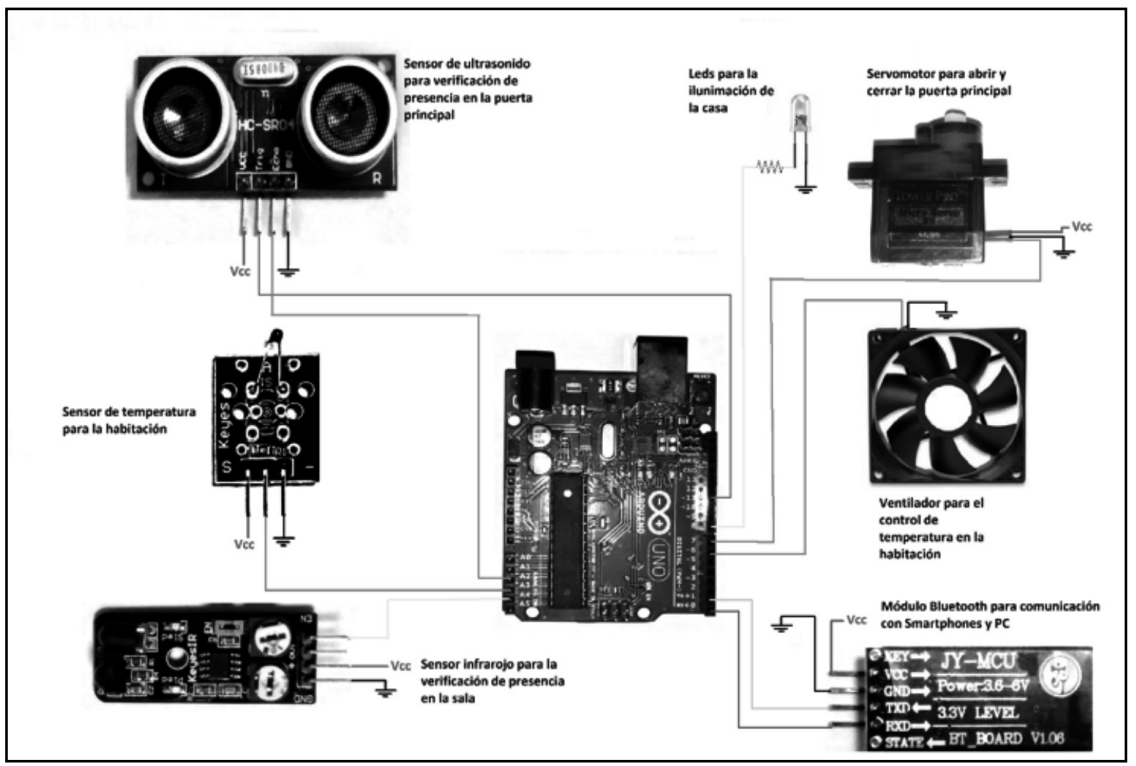

Fuente. Elaboración propia de los autores.

Figura 3. Ubicación del sensor de ultrasonido en la casa

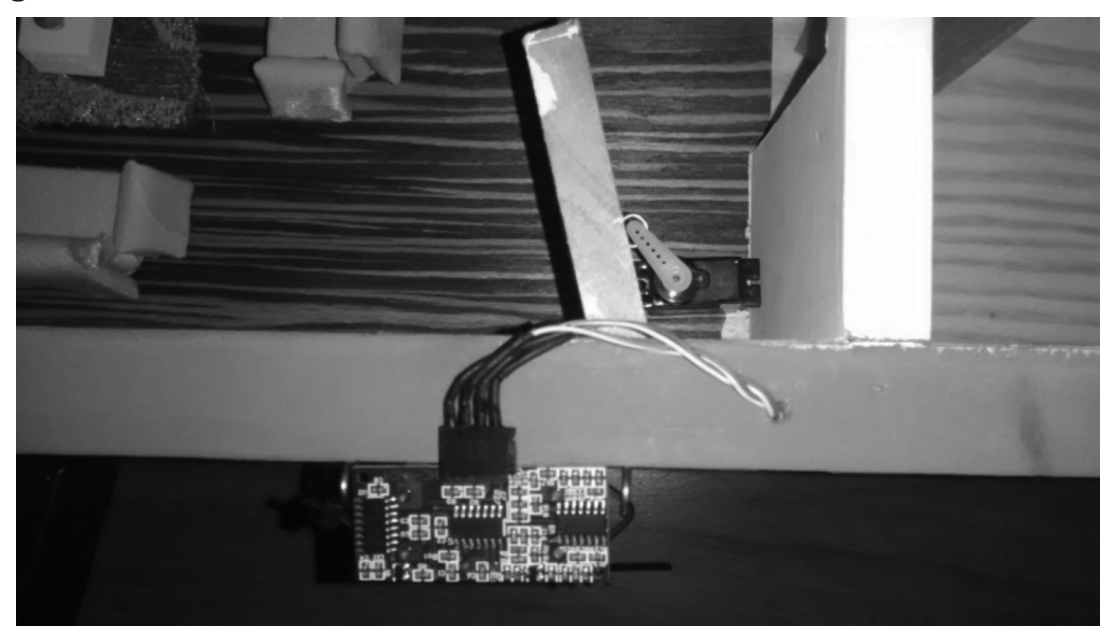

Fuente. Elaboración propia de los autores. 


\section{Conclusiones}

El logro principal de este proyecto fue construir un prototipo funcional de la maqueta de una casa con elementos domóticos, que puede ser controlada por dispositivos móviles. También, se definió una metodología clara relacionada con herramientas utilizadas de manera general, que permitirían realizar prototipos con las mismas características y con equipos de trabajo que estén en una situación parecida.

Otro de los grandes logros del proyecto, fue organizar un equipo de trabajo conformado por varias personas con diferentes niveles de experiencia en temas relacionados con la domótica: electrónica, mecánica y programación. A pesar de tener un equipo variado, tanto en niveles como en disciplinas, con esta metodología se logró desarrollar el prototipo funcional, de la misma forma como lo haría un equipo multidisciplinario en un escenario de producción tradicional.

Con base en esta experiencia, es necesario concluir que el desarrollo de este tipo de proyectos requiere de un trabajo considerable en diferentes áreas; no solamente en electrónica, mecánica y programación, sino que además entran factores estéticos, manejo de herramientas CAD para simulación, etc. Es muy valioso para el proyecto contar con integrantes que tengan conocimientos en otras áreas, pues el producto final resulta muchísimo más valioso y vistoso para el observador.

A partir de los resultados obtenidos de este prototipo, surgen nuevos proyectos para la casa inteligente que permitirán poner en práctica los diversos conceptos aprendidos y asimilar varios experimentos domóticos interesantes. Entre estos proyectos se propone emprender los siguientes:

- Utilizar el módulo Bluetooth para obtener información de los diversos sensores que hacen parte de la "Casa inteligente". De esa manera, será posible obtener y monitorear estos valores desde un teléfono inteligente y/o un computador. 
- Visualizar los valores de los sensores en Internet, con el fin de monitorear la información captada por los diversos sensores distribuidos por la casa desde cualquier computador conectado a Internet. Esto implicará transferir periódicamente la información de los sensores a un servidor Web de forma tal que se pueda obtener esta información desde un "Navegador Web".

- Detectores de fugas de gases y agua.

- Alarma ante incendios.

- Un módulo RFID para mejorar la seguridad de la casa, para que solo aquellos autorizados puedan ingresar a ella.

Finalmente, el uso de una metodología clara y de herramientas de tipo libre hacen posible que el proyecto pueda expandirse fácilmente, a través de extensiones o componentes. 


\section{Referencias bibliográficas}

Brush, A. B., Lee, B., Mahajan, R., Agarwal, S., Saroiu, S., \& Dixon, C. (2011). Home automation in the wild: challenges and opportunities. Proceedings of the SIGCHI Conference on Human Factors in Computing Systems (CHI ‘11). New York, NY: ACM.

Gauger, M., Minder, D., Marrón, P. J., Wacker, A., \& Lachenmann, A. (2008). Prototyping sensor-actuator networks for home automation. Proceedings of the workshop on Real-world wireless sensor networks. New York: ACM, pp 56-60.

Irwin, D., Barker, S., Mishra, A., Shenoy, P., Wu, A., \& Albrecht, J. (2011). Exploiting home automation protocols for load monitoring in smart buildings. Proceedings of the Third ACM Workshop on Embedded Sensing Systems for Energy-Efficiency in Buildings. New York: ACM, pp. 7-12.

Jacobson, I., Booch, G., \& Rumbaugh, J. (1999). The Unified Software Development Process. New York: Addison-Wesley Professional.

Mateos, F., González, V. M., Poo, R., García, M., \& Olaiz, R. (2010). Design and Development of An Automatic Small-Scale House For Teaching Domotics. Proceedings of the Frontiers in Education Conference, 2010. On 31st Annual - Volume 01. Washington, DC, USA: IEEE Computer Society, pp T3c-1-5.

McRoberts, M. (2013). Beginning Arduino. (s.c) Apress.

Randall, D. (2003). Living Inside a Smart Home: A Case Study. En Inside the Smart Home. Springer, Heidelberg, pp. 227-246.

Rodden, T., \& Benford, S. (2003). The evolution of buildings and implications for the design of ubiquitous domestic environments. Proceedings of the SIGCHI Conference on Human Factors in Computing Systems. New York: ACM, pp. 9-16. 
Rode, J., Toye, E., \& Blackwell, A. (2005). The Domestic Economy: a Broader Unit of Analysis for End User Programming. Proceedings of the SIGCHI Conference on Human Factors in Computing Systems. New York: ACM, pp. 1757-1760.

Vassev, E., Hinchey, M., \& Nixon, P. (2010). Prototyping home automation wireless sensor networks with ASSL. Proceedings of the 7th international conference on Autonomic computing. New York: ACM, pp. 71-72.

Woodruff, A., Augustin, S., \& Foucault, B. (2007). Sabbath Day Home Automation: "It's Like Mixing Technology and Religion". Proceedings of the SIGCHI Conference on Human Factors in Computing Systems. New York: ACM, pp. 527-536. 